\title{
The Supersti+Tech Project: A Reinterpretation of Superstition through New Media Technology
}

\author{
Jinhee Kim \\ Aalto University, School of Arts, Design and Architecture \\ Hämeentie 135, 00560 Helsinki, Finland \\ jin.kim@aalto.fi
}

\begin{abstract}
"Supersti+Tech" is a compound word of "Superstition" and "Technology". The purpose of this project is to reinterpret superstition with new media devices through which juxtaposes and combines various paradoxical concepts such as irrational versus rational, fiction versus fact, and virtual versus real. The outcome of this project displays three interactive installations which use computer processing and mechanical engineering: A Fortune-Telling Bird, A Lucky Charm Drawing Robot, and Plastic Divination. In 2014, A Fortune-Telling Bird was exhibited at Live Herring '14 in Jyväskylä, Finland and A Lucky Charm Drawing Robot was exhibited in Helsinki, Finland. The production of Plastic Divination is in progress
\end{abstract}

Superstition. Media art. New media technology. Interactive installations.

\section{INTRODUCTION}

Throughout human history, superstition has been found in almost every culture in different forms and methods and has played various roles in people's daily lives (cf., Flower 2007, Johnston 2009, Lawson 1910, Sibylla 2009). Even though we are living in the "modernized" 21st Century enjoying advanced technologies more than ever before, interestingly, many of us still often engage in superstitious practices to relieve anxiety from uncertainty of our lives and the future. For example, a four-leaf clover bringing good luck and the fear of Friday the 13th are common myths widely believed in many cultures (Sibylla 2009). In addition, superstition is now successfully adapting its form to new media technologies, so you can easily find numerous Web and smartphone applications regarding fortune-telling such as Tarot, I Ching, and Horoscope.

The Supersti+Tech project focuses its attention on the fact that superstition has its own unique roles and territory in this technology-centred era, of which science and logical reasoning can never replace. This project tries to explore this irony between superstition and technology with new media art using computer processing and mechanical engineering. The art installations of this project gather information from participants and then give answers based on a set of rules and random data. Although the way to engage in superstitious practices with the art installations is different from the traditional ones, the content and how the mechanism of superstition works are not much changed - it basically appeals to people's wishes and fears. This project reinterprets superstition with new media devices through which juxtaposes and combines various paradoxical concepts such as irrational versus rational, fiction versus fact, and virtual versus real and further provides integrative and interactive media experiences to participating subjects.

\section{THE SUPERSTI+TECH PROJECT}

The ultimate outcome of this project displays a series of three interactive installations: $A$ FortuneTelling Bird, A Lucky Charm Drawing Robot, and Plastic Divination. The concept, inspirational backgrounds, production, and participation processes of each installation are explained in detail in this section.

\subsection{A Fortune-Telling Bird}

\subsubsection{The Concept and Background}

A Fortune-Telling Bird is the first product of the Supersti+Tech Project and it is an interactive installation providing its participant's fortune via Twitter. 
The idea of $A$ Fortune-Telling Bird originates from the traditional Chinese bird fortune-telling which predicts one's fortune from a card randomly picked by a bird (Gray 1878). The technique of traditional bird fortune-telling can vary by individual fortunetellers. In general, a client asks his or her fortune regarding love, work, health, or the anticipation of the upcoming year to a fortune-teller, and then the fortune-teller shakes a box containing a lot of fortune papers and places it in front of his or her trained bird's cage. When the fortune-teller opens the door of the cage, the bird comes out and picks up one or several papers randomly, on which the client's fortune is written.

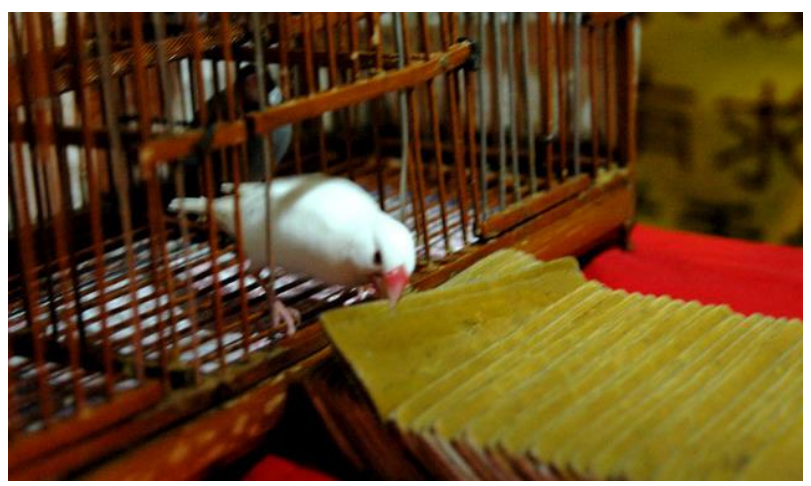

Figure 1: A traditional bird fortune-telling (retrieved from http://asiaobscura.com/2011/01/what-the-fortune-tellertold-me-hong-kong.html)

\subsubsection{The Installation}

In my project, a fortune-telling bird is replaced with a robotic bird sitting inside a wooden cube device which has the length, width and height of 25 centimetres (see Figure 2). The device consists of two servo motors, a coin acceptor, a thermal printer, and a motorised slider operated by mechanical engineering and computer coding. On the front side of the cube, there are a big hole for the bird to come out, a coin slot on the top-right side, and a printer slot on the bottom-right side. A decorative round wooden board is attached in front of the hole.

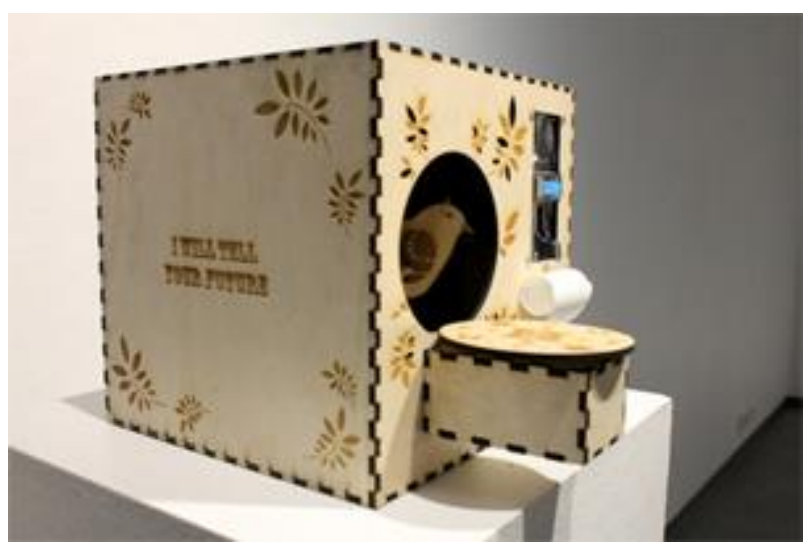

Figure 2: A Fortune-Telling Bird

The device works as follows: (i) A participant inserts a coin into the coin slot.

(ii) A Fortune-Telling Bird begins to come out of the device, and the round board starts to spin at the same time.

(iii) The board stops spinning when the bird arrives at the front part of the board.

(iv) The bird bends down and picks a random point within the pattern of the board, and then a thermal printer starts to print out the participant's fortune paper.

(v) The bird returns to its place.

(vi) The participant can take his or her fortune paper by cutting it with scissors.

When a participant comes to the device, there is an instruction next to the device about the participation procedures. A participant is required to ask a specific question in his or her mind before putting a coin into the coin slot. The coin acceptor is programmed to identify $10,20,50$ cents, and 1 euro for the participants' convenience. If the sensor in the coin acceptor detects one of those four coins, it activates two motors simultaneously: a motorized slider of the bird and a servo motor of the round board. A motorized slider holding the wooden bird moves the bird forward to the front of the board, and at the same time, a servo motor supporting the round board spins either clockwise or counterclockwise random times to prevent the bird from picking the same pattern. The servo motor of the round board stops spinning when the bird arrives at the front part of the board, and another servo motor adhered to the bird's body rotates about 90 degree angle clockwise so that the bird can pick a random point of the pattern on the board.

Finally, the thermal printer starts printing a paper which has a random tweet taken from real-time Twitter based on the following fourteen fortunerelated English keywords: good, bad, happy, sad, life, love, work, health, money, luck, future, god, chance, and destiny. When all works are done, the participant can take his or her fortune paper by cutting it with scissors.

For a keyword search mechanism, A FortuneTelling Bird uses a PHP script derived from a website, Månsteri (see http://mansteri.com). This PHP script uses the Twitter REST API version 1.1 (see https://dev.twitter.com) to search for keywords. The Arduino code uses the Eternet shield to read the search results from the website.

\subsubsection{Relation to Twitter}

As one of the most popular social networking services, Twitter is the key ingredient in A Fortune- 
Telling Bird. One hundred and forty-character messages called "tweets" are sent and read via Twitter website, mobile device, or SMS (Short Message Service). There are 288 million monthly active users and 500 million daily tweets in Twitter (see https://about.twitter.com). A Fortune-Telling Bird randomly chooses a real-time tweet from Twitter and prints it out to the participants. The participants get the answer to their question through this computer algorithm, which works logically but is much more random, unpredictable, and interactive than the traditional way of bird fortune-telling.

\subsubsection{The Exhibition and Participant Reactions}

A Fortune-Telling Bird was shown at three different places in Finland - at Aalto Innovation House in Espoo (2013), Live Herring'14 at Gallery Ratamo in Jyväskylä (2014), and Kaapeli in Helsinki (2014).

The exhibition invited by Live Herring, which is the association promoting media arts and media culture, was held for about two months in Jyväskylä, Finland. In that exhibition, all the participants of $A$ Fortune-Telling Bird were encouraged to pin their fortune papers on the wall after having their fortune told (see Figure $3 \& 4$ ). The information that the fortune paper was retrieved from Twitter was noticed to the participants.

Approximately two hundred fortune papers were posted on the wall on the last day of the Live Herring'14 exhibition, and visitors were able to browse diverse answers to the participants' questions on the wall. Some of the answers from real-time tweets were insightful and poetic just like the real divination from human fortune-tellers. The followings are the examples:

- "Love doesn't hurt, loving the wrong person does."

- "People just don't want you to be happy."

- " "I rather get money."

- "Someone gives me some good luck."

- "Life is too precious to waste your time thinking about someone who doesn't treat you right."

I randomly chose sixteen out of all participants and interviewed them by asking whether they felt the fortune papers given by the bird really answered their questions and whether the fact that their fortune actually came from Twitter had affected on their belief.

Eleven participants responded that they felt they had gotten the right answer from the bird and didn't think that the fact that their fortune came from Twitter had diminished the authenticity of the fortune content. One participant reported her feeling about the fortune paper as follows:

I don't care where the answer came from as it was quite scary to get such an accurate reply to my quite specific question. I am not superstitious person at all, but this was the first time I thought I should be at least a bit. Or maybe it was one of these coincidences. I never won in lottery but got the perfect answer from the bird.

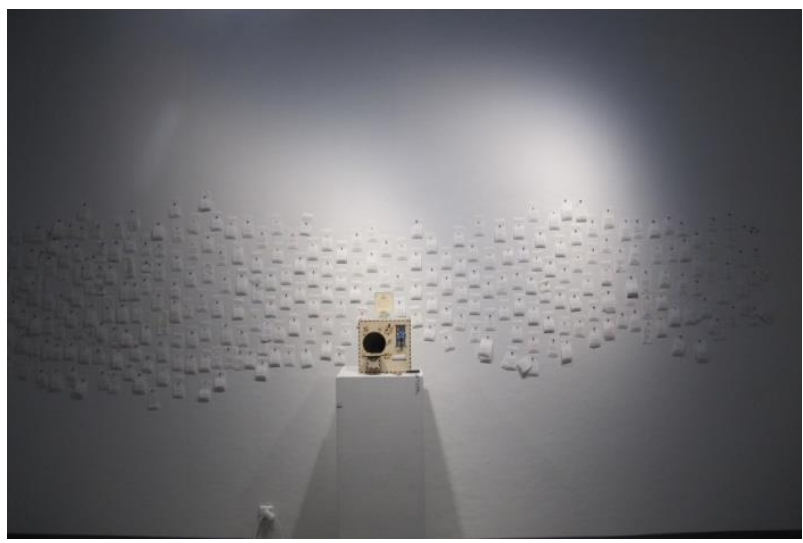

Figure 3: Exhibition at Gallery Ratamo, 2014, Finland

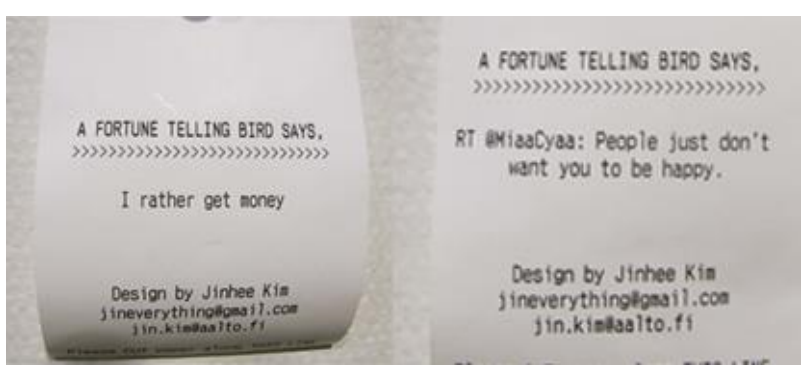

Figure 4: The printed fortunes: "I rather get money (left)" and "People just don't want you to be happy (right)," exhibited at Gallery Ratamo, Finland, 2014

\subsection{A Lucky Charm Drawing Robot}

\subsubsection{The Concept and Background}

A Lucky Charm Drawing Robot is the second outcome of Supersti+Tech Project, which is an interactive installation drawing a lucky charm paper after reading a participant's palm. A Lucky Charm Drawing Robot is a combination of three traditional superstitious beliefs: lucky charm papers, palmistry, and four-leaf clover.

In the East Asia, lucky charm papers have been believed to protect their careers from evil forces or bring good luck (Baker 2008, Sibylla 2009). A traditional form of lucky charm papers includes Chinese character phrases or abstract sketches of spiritual forces (Baker 2008). The sketches are drawn with red pigments ground from Cinnabar, which symbolises power to drive away evil spirits, on a yellow paper dyed with the flowers of Chinese Scholar Tree (Oh 2003) (see Figure 5). 


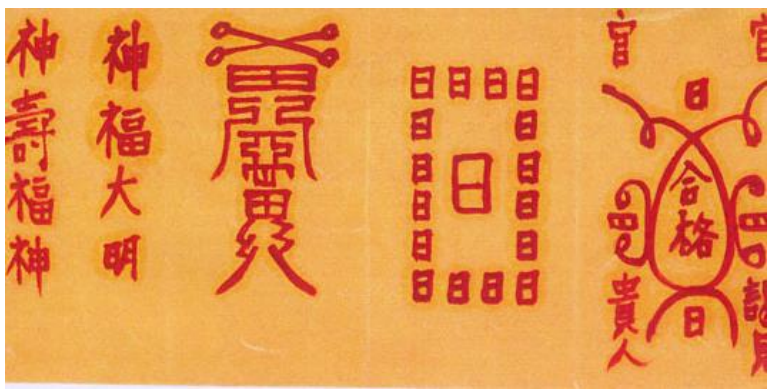

Figure 5: Traditional lucky charm paper (retrieved from http://blog.naver.com/sajuboa/70151201901)

Palmistry has been practiced since ancient times. A palmist predicts one's character and future by examining the lines or height of the palm of their hand. In classical palmistry, four major lines; heart line, head line, fate line and life line; are regarded as important (Walter 2000) (see Figure 6).

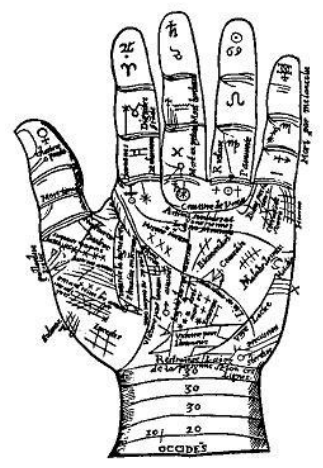

Figure 6: Palm reading (retrieved from http://www.psychic-side.com)

As one of lucky charms, Four-leaf clover is considered as a symbol of good luck and good fortune because it is rarer or harder to find than a three-leaf clover. The verdant colour of the four-leaf clover also represents the essence of life (Sibylla 2009).

\subsubsection{Installation}

A Lucky Charm Drawing Robot consists of two boxes. The right box is for reading the participant's palm, which has a GSR (Galvanic Skin Response) reader with two copper boards and a LED light. The left box is for drawing a lucky charm paper, which has robot arms made up of three servo motors and a light sensor in the middle of the square frame of the box (see Figure $7 \& 8$ ).

There is a small box for yellow papers next to the device and also two stamps engraved with a four-leaf clover. The robot arms of the left box are holding a red brush pen corresponding to the red pigments ground from Cinnabar in the traditional lucky charm paper. The yellow paper also represents the traditional yellow paper dyed with the flowers of Chinese Scholar Tree.

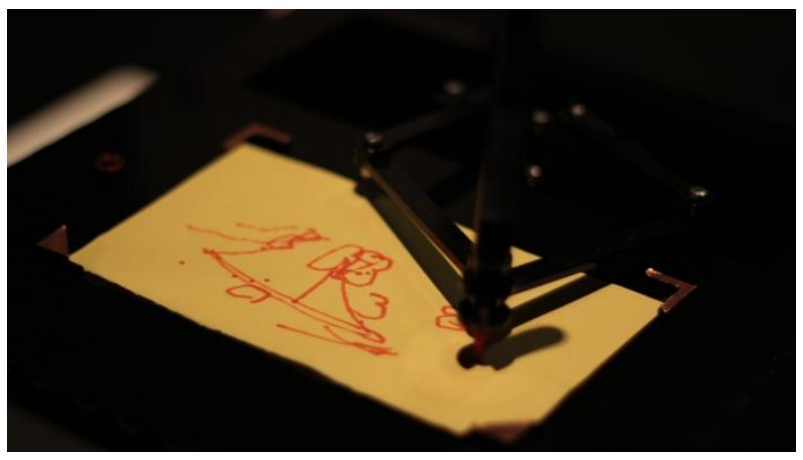

Figure 7: The left box equipped with robot arms has a small hole in the middle of the square frame of the box and a small round holder next to the frame for a brush pen

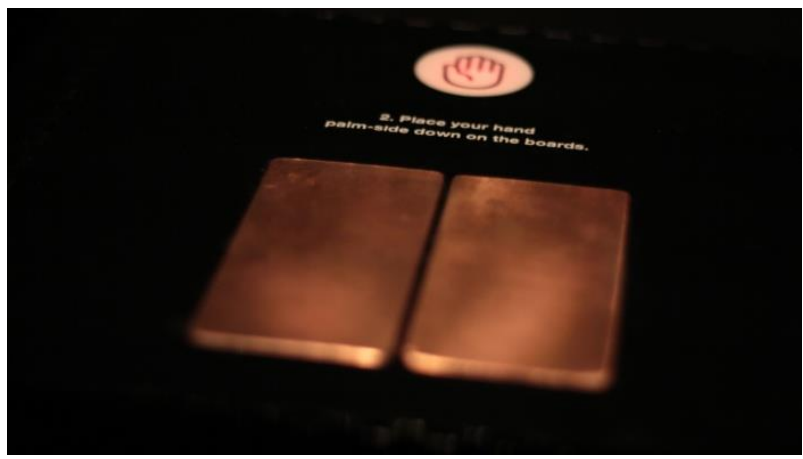

Figure 8: On the top side of the right box, there are two copper boards (a GSR reader) and a LED lamp

The device works as follows:

(i) A participant puts a yellow paper in a square frame of the left box and places his or her hand on two copper boards of the right box.

(ii) A LED lamp of the right box changes its colour from red to green and blinks while the device reads the participant's palm.

(iii) Robot arms of the left box draw a random sketch with a red brush-pen on the yellow paper.

(iv) The LED lamp changes its colour from green to blue after the drawing is complete.

(v) A participant takes the charm paper out from the frame and decorates his or her own charm by stamping two seals on it. The seals are engraved with a four-leaf clover.

Participants are required to follow the instruction regarding the procedures to get the lucky charm. When a participant puts a paper of 15 by 8.5 centimetres $(15 \times 8.5 \mathrm{~cm})$ in the frame of the left box and places his or her hand on the copper boards of the right box, the light sensor underneath the small hole of the left box detects the brightness level declining. Then the GSR reader on the right box measures the participant's palm conductivity. The robot arms start drawing only if the brightness level of the light sensor and the conductivity level 
of the participant's palm are satisfied with the specific condition.

If both conditions are met, the LED lamp changes its colour from red to green and starts blinking, and then three servo motors of the robot arms start drawing a sketch with a red brush pen on a yellow paper. The participant takes out the paper from the frame after the drawing is finished. The participants can decorate the charm paper with a four-leaf clover seals and carry it with them all the time.

The idea of the robot arms is inspired by Plotclock (see https://github.com/9a/plotclock), which is a small robot showing the time by drawing numbers on the board. The Arduino code of Plotclock version 1.0 has been modified to create various and random sketches of the charm papers. Not only the numbers by Plotclock but also the rectangle, square, ellipse, circle, lines and dots are designed with the Arduino code for this project (see Figure 9). There are ten different sets of codes working based on the level of the participant's palm conductivity. However, no participant has the same design as others' since each set coded with random numbers ends up with different results every time.

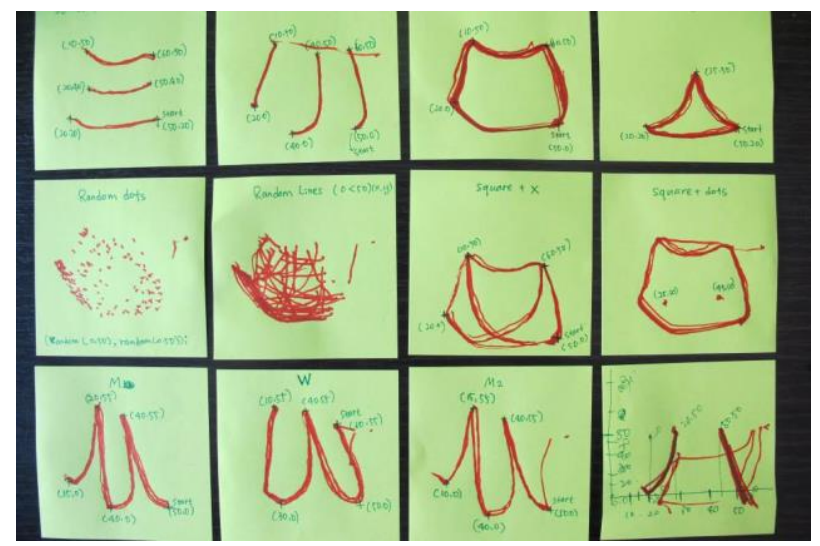

Figure 9: Diverse basic patterns with the Arduino code

The participants can also decorate a more unique lucky charm paper by stamping a seal on it, which is engraved with a four-leaf clover. They are also requested to carry the charm paper always with them to bring good luck as well as to ward off evils (see Figure 10).

\subsubsection{Relation to a GSR Reader}

A Galvanic Skin Response (GSR) reader is a method of measuring the skin's resistance to an electrical current. The electrical conductance between two points on the skin is increased by the moisture on the skin. A low GSR shows tension, and a high GSR shows relaxation. A GSR reader is often used in polygraph (lie director) tests since skin conductance is an instant and direct measure of emotional and sympathetic responses (Mason 2001).

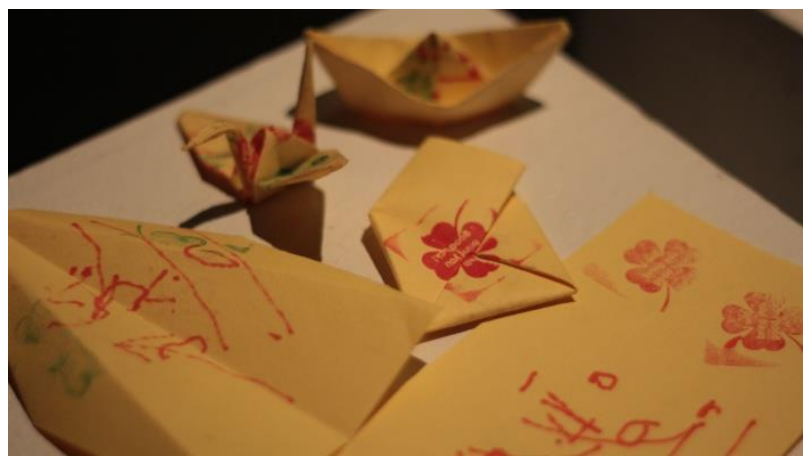

Figure 10: Lucky charms decorated by the participants

A Lucky Charm Drawing Robot uses two copper boards as a GSR reader which measures the electrical conductance of the participant's palm. While a participant places his or her hand on the GSR reader, the LED lamp blinks as if the device is reading the participant's palm. The GSR reader of A Lucky Charm Drawing Robot represents a palmist who reads the clients' palm and decides what kind of charm papers would be the best for the client.

\subsubsection{The Exhibition and Participant Reactions}

A Lucky Charm Drawing Robot was shown at Kaapeli, Helsinki in 2014. The robot had drawn various sketches with random numbers, lines, dots and shapes that looked like abstract drawings (see Figure 11).

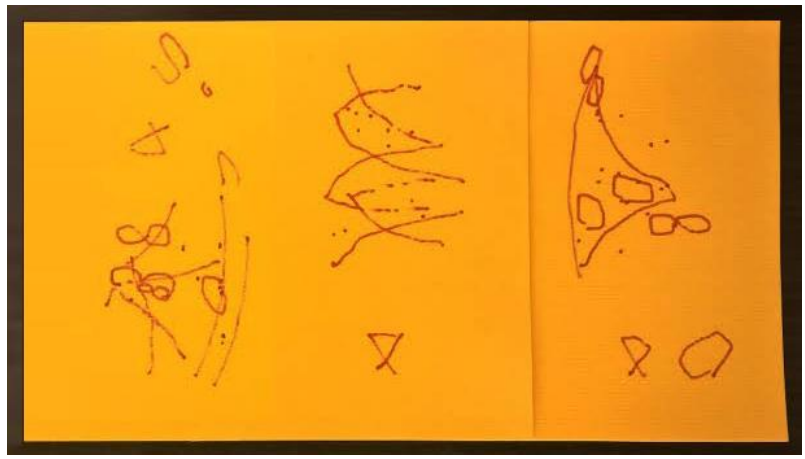

Figure 11: Sketches with random numbers, lines, dots and shapes

I randomly chose twenty-two participants to hear their opinions and experiences about $A$ Lucky Charm Drawing Robot. Five out of twenty-two interviewees said that they interpreted the numbers on the charm paper as lottery numbers, and eighteen interviewees thought that the sketches on the charm papers might have some meanings.

At the exhibition in Kappeli, both $A$ Fortune-Telling bird and A Lucky Charm Drawing Robot were 
shown at the same place, so I was able to interview the participants who had experienced both devices. One of my questions was "Which device was more arousing your superstitious belief?" and one participant answered as follows:

\begin{abstract}
I think the lucky charm paper is more human because of its hand-drawn-like. There is something fascinating when pen touches paper and makes lines. It is one kind of human communication, and handwriting tells so much about the writer. In that sense the lucky charm paper is more "real" to me, so it is more superstitious.
\end{abstract}

On the contrary, another participant answered that A Fortune-Telling Bird was more superstitious:

\begin{abstract}
The fortune-telling paper has more resonant as it's text-based; the lucky charm paper is a lot more abstract and hence more difficult to relate to it. But I like the idea of the lucky charm drawing robot's output being based on the actual physical reading of your palm - it feels more personal and not just some random tricks. But the fortune-telling paper has more "superstitious" effect on me, I might actually follow some of its "advice" if I found it relevant.
\end{abstract}

\subsection{A Plastic Divination}

The third product of Supersti+tech Project will be Plastic Divination. Since the production of Plastic Divination is still in progress, only its concept and inspirational background are briefly mentioned here.

The basic idea of Plastic Divination is inspired by Molybdomancy (cf., Wynn, Wiggins, \& Harris 2001), a technique of divination using molten metal. In my project, Plastic Divination will be a 3D printer that creates 3D sculptures based on mechanical engineering and computer programming. When a participant puts a horseshoe-shaped plastic into the 3D printer device, which is specially designed for this project, a random shaped 3D sculpture will be produced. The resulting shape can be either directly interpreted as a sign for the participant's future or shown in candlelight to interpret the shapes of its shadow like the traditional way of Molybdomancy.

\section{DISCUSSION}

The most interesting finding from this project for me was the participants' interpretation of artworks. Most participants whom I interviewed were highly engaged in their fortune-telling papers although they knew that the answers had come from computer programming. At the exhibitions of $A$ Fortune-Telling Bird, some participants tried hard to discover the relevance between their questions and the answers from Twitter. For example, a short tweet promoting a musician's new album has become a specific message to one's question. Also, A Lucky Charm Drawing Robot's abstract patterns with squares, dots and several numbers on a yellow paper turned out to be significant figures bringing good luck to the participant. The participants seemed to try to either find the meaning behind the patterns or assign a certain meaning to them. One participant stated:

\section{The patterns of A Lucky Charm Drawing Robot were quite abstract with some number-like figures. But the abstract shapes instantly reminded me of faces, animals and so on, and with numbers, my mind started to create a story.}

These reactions can be explained by the Forer Effect theory in psychology. The theory refers to the tendency that people accept general and vague statement about their personality as special to them although it applies to a wide range of people (Forer 1949). As a related phenomenon, subjective validation refers to any situation where two irrelevant events are perceived as having some hidden relationship because of the observer's prior belief or the expectations that such correlations must exist (French \& Ston 2013). These theories explain why people tend to engage in superstitious practices such as fortune telling, astrology, and biorhythms and give us an insight to understand the way the participants interact with my art installations.

A Fortune-Telling Bird and A Lucky Charm Drawing Robot are devices that work with scientific, technological, logical, and factual methods operated by computer processing and mechanical engineering. However, the randomness of the system, such as a real-time tweet randomly taken out from Twitter and the coding with random numbers, results in unpredictable and uncertain outcomes. I observe that the participants interpret this randomness as a meaningful story by linking it to their wishes and fears, which is a totally unscientific, irrational and fictional behavior. Perhaps, in the end, the one who plays the major role of a fortune-teller is neither the bird nor the robot but the participant himself or herself.

\section{REFERENCES}

Baker, D. (2008) Korean Spirituality. University of Hawaii Press, HI.

Flower, M.(2017) Seer in Ancient Greece. University of California Press, CA.

Forer, B. R. (1949) The fallacy of personal validation: A classroom demonstration of gullibility. Journal of Abnormal and Social Psychology, 44, 118-123. 
French, C. C. \& Ston, A. (2013) Anomalistic Psychology: Exploring Paranormal Belief and Experience. Palgrave Macmillan, UK.

Gray, J. H. (1878) China: A History of the Laws, Manners and Customs of the People. Macmillan, London.

Johnston, S. (2009) Ancient Greek Divination. Wiley-Blackwell, New Jersey.

Lawson, J. C. (1910) Modern Greek folklore and ancient Greek religion: A study in survivals. Cambridge University Press, Cambridge.

Mason, L. J. (2001) Guide to Stress Reduction, 2nd Ed. Celestial Arts, CA.
Oh, H. (2003) Bujukdaesajun [The Unabridged Dictionary of Talisman]. Donghaksa, Seoul.

Sibylla, Putzi-O. (2009) $A$ to $Z$ world superstitions and folklore 175 countries: Spirit worship, curses, mystical characters, folk tales, burial and the dead, animals, food, marriage, good luck, bad luck, totems and amulets and ancestor spirits. World Trade Press, CA.

Walter, G. (2000) Das neue Lexikon des Aberglaubens [The New Encyclopedia of Superstition]. Piper, Munich.

Wynn, C. M., Wiggins, A. W., \& Harris, S. (2001) Quantum Leaps in the Wrong Direction: Where Real Science Ends and Pseudoscience Begins. Joseph Henry Press, USA. 